\title{
Outbreak Management Scenario in Senior's Residences
}

\author{
Benoit Samson*
}

Anesthesiologist (RTD), LCOL (RTD) Canadian Medical Corps, Canada

\section{Introduction}

The yellow and black checkered colours of quarantine, like those hoisted recently on the masts of the Diamond Princess on February 3 in Yokohama, the Grand Princess in California, the Zaandam in Fort Lauderdale, and on more than 25 other cruise ships worldwide since February 2020, should perhaps also be floating over some seniors' residences. The time has come to consider treating the senior "passengers of our land ships" the same way we did with cruise passengers in distress. Currently, we appear to be making the same mistakes in the management of our elderly that were made with cruise ship passengers just a few weeks previously.

\section{Cruise Ship Experience}

In February 2020, Public Health authorities in Japan were the first to decide that cruise ship passengers infected or carriers of COVID-19 had to be quarantined on the ship. They did so with the consent of health authorities in many other countries who in fact soon had to do the same. Logically, they opted for the classic multi-centenary use of quarantine for any ship approaching our shores with passengers who could transmit their diseases to our populations. There were many valid historical arguments supporting that decision. Like so often employed in the past, it was believed that a quarantine that confined passengers to their state rooms would stop the spread of the disease until such time as the sick were cured, thus allowing everyone on board to disembark safely.

However, even sheltered in their most modern state rooms and away from each other on the Diamond Princess, it rapidly became evident that it was impossible to control the infections. From only a few passengers at the start of the quarantine, 700 became quickly infected and several died. Furthermore, health workers called in as reinforcements became sick themselves and this despite having the skills to work in contaminated environments. It rapidly became obvious that the management strategy to control such COVID-19 infestations had to be different than the usual maritime quarantine solutions for this predicament. Once it was realized that the cruise ships were excellent incubation and hatching environments that were impossible to control, instead of keeping the passengers confined, the management strategy changed to quickly evacuating the passengers. Those who tested negative for COVID-19 were transferred to clean land facilities for quarantine while those who tested positive were sent to hospitals for treatment. For that group of individuals, this was the solution to effectively decrease the transmission and deaths from COVID-19.

\section{Nursing Homes and Long-Term Care Facilities}

There are hundreds of thousands of "homes" for seniors across the world. They vary in size, scope and level of care offered to their residents. The quality and adaptability of the premises and personnel also differs significantly from place to place. The possibility of safely-separating hot and cold zones should an outbreak of infections occur in these facilities is also extremely variable.

During the first four months of 2020, nursing homes and long-term care facilities have experienced the worst incidence of deaths from COVID-19 infections. In Canada, approximately $50 \%$ of all deaths from COVID-19 occurred in long-term care facilities. In the United States the incidence is uncertain since that data is not collected nationwide but is assumed to be over $50 \%$ of all casualties. However, the data is available in some states: Pennsylvania reports an incidence of $80 \%$ and Connecticut, approximately $90 \%$. The same horrible mortality data is evident worldwide and results from the current faulty management strategies for seniors lodged in certain longterm care facilities.

As was the case on cruise ships, whenever an outbreak of COVID-19 occurs in some of our long-term care facilities, it is only a matter of time before the contagion spreads exponentially to vulnerable residents and workers alike. Fortunately, some residences are better equipped in personnel, confinement procedures and with modern construction standards to effectively institute hot and cold zones within their walls with minimal or no cross contamination. The percentage of seniors' residences which are able to properly selfmanage outbreaks of COVID-19 is unknown. However, anecdotal evidence seems to indicate that most can prevent or contain such outbreaks.

*Corresponding author: Benoit Samson, Anesthesiologist (RTD), LCOL (RTD) Canadian Medical Corps, 2143 Dutton Crescent, Ottawa, Ontario K1J 6K3, Canada, Tel: 613-748-9684

Accepted: May 28, 2020

Published online: May 30, 2020

Citation: Samson B (2020) Outbreak Management Scenario in Senior's Residences. Res Rev Infect Dis 3(1):64-65 
Our efforts to prevent the high mortality rate experienced in long-term care facilities should therefore be concentrated on those facilities that, for whatever reason can manage such outbreaks themselves. These efforts should be deployed early and aggressively to save lives since it is now known that COVID-19 is very contagious and deadly for the residents of seniors' homes.

The time has come to apply the knowledge acquired over the past four months in the management of COVID-19 outbreaks in the confines of cruise ships to the long-term care facilities that are unable, for whatever reason, to cope with a COVID-19 outbreak within their walls. "The knowledge accumulated in the analysis of this pandemic is crucial for decision-making in the management of this infestation". a

When such an outbreak occurs, the same management strategy used successfully for cruise ships should be implemented urgently to save the lives of senior residents. In the urgent phase one of this proposed scenario, all the residents and personnel of infested facilities unable for whatever reason to control the infestation should rapidly be evacuated temporarily from the premises while a thorough disinfection of the building is carried out. As was done with cruise-ship passengers, the residents and employees testing positive to COVID-19 should be transferred to a COVID-19 hospital for treatment. If such a dedicated hospital is not available, the Red Cross can rapidly and effectively install mobile treatment facilities for that purpose anywhere in America. The residents

a Nature. Volume 579 Issue 7800, 26 March 2020. and employees testing negative should be transferred to a clean facility to receive adequate care tailored to their needs. The use of hotels to relocate residents may not be possible since most of the residents of nursing homes and long-term care facilities are not autonomous enough to care for their basic needs. Residents that are autonomous enough could be relocated temporarily in hotels while their residence is decontaminated.

In phase two, all residents and employees of the quarantined premises could return to their long-term care facilities under new strict protocols devised during the lock down. Any and all individuals allowed to enter the disinfected premises would first have to test negative for COVID-19. Strict sanitary protocols should be implemented. Any individual allowed to enter the premises after the reopening would have to test negative for COVID-19. All personnel would wear masks until the pandemic is over. The staff and residents that tested positive would not be allowed to return to their original home until they test negative.

Only when the lessons learned from recent history are applied will the deadly cycle of cross contamination be broken and lives otherwise lost in our long-term care facilities saved.

Admittedly, this is not an easy scenario to implement, but everything that seemed easy and potentially effective has been tried with disastrous results to date. The time has come to find a pragmatic out-of-the-box solution to this urgent problem, using lessons learned recently from the cruise industry to effectively manage this unprecedented humanitarian crisis for our elderly.

DOI: $10.36959 / 719 / 566$

Copyright: (C) 2020 Samson B. This is an open-access article distributed under the terms of the Creative Commons Attribution License, which permits unrestricted use, distribution, and reproduction in any medium, provided the original author and source are credited. 